\title{
Re-excision or "wait and watch"-a prediction model in breast phyllodes tumors after surgery
}

\author{
Xue Chao ${ }^{1,2,3}$, Xiaoyan Jin ${ }^{1,4 \#}$, Cui Tan ${ }^{5}$, Peng Sun ${ }^{3}$, Junwei Cui ${ }^{6}$, Hui Hu ${ }^{6}$, Qian Ouyang ${ }^{1,2}$, Kai Chen ${ }^{1,2}$, \\ Wei $\mathrm{Wu}^{1,2}$, Zhanghai $\mathrm{He}^{5}$, Yan $\mathrm{Nie}^{1,2}$, Herui Yao ${ }^{1,2}$ \\ ${ }^{1}$ Guangdong Provincial Key Laboratory of Malignant Tumor Epigenetics and Gene Regulation, ${ }^{2}$ Breast Tumor Center, Sun Yat-sen Memorial \\ Hospital, Sun Yat-sen University, Guangzhou 510120, China; ${ }^{3}$ Pathology Department, Sun Yat-sen University Cancer Center, Sun Yat-sen \\ University, Guangzhou 510000, China; ${ }^{4}$ General Department, ${ }^{5}$ Pathology Department, Sun Yat-sen Memorial Hospital, Sun Yat-sen University, \\ Guangzhou 510120, China; ${ }^{6}$ Department of Breast Surgery, Peking University Shenzhen Hospital, Shenzhen 518036, China \\ Contributions: (I) Conception and design: H Yao, Y Nie; (II) Administrative support: H Yao, Y Nie; (III) Provision of study materials or patients: Cui \\ Tan, P Sun, H Hu, Z He, J Cui; (IV) Collection and assembly of data: Q Ouyang, K Chen, W Wu; (V) Data analysis and interpretation: X Chao, X \\ Jin; (VI) Manuscript writing: All authors; (VII) Final approval of manuscript: All authors. \\ "These authors contributed equally to this work. \\ Correspondence to: Herui Yao, MD, PhD. Breast Tumor Center and Department of Oncology, Sun Yat-sen Memorial Hospital, Sun Yat-sen University, \\ 107 Yanjiang West Road, Guangzhou 510120, China. Email: yaoherui@163.com; Yan Nie, MD, PhD. Breast Tumor Center, Sun Yat-sen Memorial \\ Hospital, Sun Yat-sen University, 107 Yanjiang West Road, Guangzhou, 510120, China. Email: nieyan7@mail.sysu.edu.cn.
}

Background: The prognosis of breast phyllodes tumors (PTs) largely depending on the pathological grading, which lacks objectivity. This study aimed to develop a nomogram based on clinicopathological features to evaluate the recurrence probability of PTs following surgery.

Methods: Data from 334 patients with breast PTs, who underwent surgical treatment at Sun Yat-sen Memorial Hospital from January 2005 to December 2014, were used to develop a prediction model. Additionally, data of 36 patients from Peking University Shenzhen Hospital (cohort 1) and data of 140 patients from Sun Yat-sen University Cancer Center (cohort 2) during the same period were used to validate the model. The medical records and tumor slides were retrospectively reviewed. The log-rank and Cox regression tests were used to develop a clinical prediction model of breast PTs. All statistical analyses were performed using R and STATA.

Results: Of all 334 patients included in the primary cohort, 224 had benign, 91 had borderline, and 19 had malignant tumors. The 1-, 3-, and 5-year recurrence-free survival was $98.5 \%, 97.9 \%$, and $96.8 \%$, respectively. Ultrasound-guided vacuum-assisted biopsy (UGVAB) is a non-inferior treatment application in benign PTs compared with open surgery [hazard ratio (HR), 2.38; 95\% confidence interval (CI), 0.59-9.58]. Width of surgical margin, mitoses, and tumor border were identified as independent risk factors for breast PTs. A nomogram was developed based on these three variables. The C-index of internal and external validation was $0.71,0.67$ (cohort 1 ) and 0.73 (cohort 2), respectively.

Conclusions: The study model presented more concise and objective variables to evaluate the recurrencefree survival of patients after surgery, which can help deciding whether to do a re-excision or "wait and watch".

Keywords: Breast phyllodes tumor; clinical prediction model; nomogram, recurrence

Submitted Nov 23, 2019. Accepted for publication Jan 03, 2020.

doi: $10.21037 /$ atm.2020.02.26

View this article at: http://dx.doi.org/10.21037/atm.2020.02.26 


\section{Introduction}

Breast phyllodes tumors (PTs) are rare fibro-epithelial tumors, the incident rate of which is less than $1 \%$ of all breast tumors (1). The World Health Organization (WHO) has divided the PTs into benign, borderline, and malignant based on the stromal cellularity, stromal atypia, stromal overgrowth, mitosis, and the characteristics of tumor border (2). However, a morphological continuity exists among PTs from benign to malignant. At present, no consensus exists among pathologists on the "cut-off" value in the grading of PTs (3). Therefore, the pathological grade of the PTs lacks objectivity.

According to previous studies, approximately $6.8-40 \%$ of patients with PTs may develop recurrence after surgery (4-7). The borderline and malignant PTs can metastasize; with the metastasis rate reaching to about $21 \%$ (8). The PTs usually metastasize via hematogenous spread and rarely via lymphatics. Furthermore, the PTs have the potential for pathological progression after recurrence. The aforementioned features indicate the importance of achieving local control in PTs.

The prognosis of PTs is mainly dependent on the pathological grade. However, the value of the pathological grade in prognosis is limited due to the subjectivity of the pathological diagnosis. Besides, PTs may have foci with benign, borderline, and malignant features in the same neoplasm. Therefore, complete excision of the tumor and multiple sampling are required for the accurate diagnosis and grading of tumors. Many retrospective studies have stated that some pathological characteristics, including hypercellularity, stromal cell atypia, mitoses, and necrosis, are correlated with the prognosis of PTs $(4,5,7,9-11)$. The role of surgical margin in the local control of the PTs was also emphasized in some studies $(7,11)$. Thus, a combination of both clinical and pathological features will lead to a more accurate evaluation of the prognosis of PTs.

A clinical predictive model can combine multiple risk factors to evaluate the prognosis of individual patients, which can also help to evaluate the interaction between risk factors. Currently, there is only one predictive model available for PT, which is published by Singapore General Hospital in 2012 (7) In this model, the criteria used included atypia, mitoses, overgrowth, surgical margin (AMOS) clinicopathological features to evaluate the recurrence-free survival of patients with PTs (7). However, the surgical margin status (positive or negative) in benign and borderline PTs were neglected in most situations, which limit the utilization of this model. Thus, due to different surgical treatments and pathological criteria involved, the same model is difficult to be applied. The present study developed and validated a clinical prediction model of breast PTs based on the retrospective data to solve this problem.

\section{Methods}

\section{Patients and study design}

This retrospective study was conducted on patients who underwent surgery for PTs from January 2005 to December 2014 at Sun Yat-sen Memorial Hospital. All patients had surgery treatment and diagnosed as PTs were included in our study. Patients with concurrent or previous malignancy, or previous history of PTs and other breast fibroepithelial tumors, or previous breast surgery, were excluded. The demographic characteristics including age, diagnosis, symptoms, present history, past history, image examination including ultrasound and mammograph results, and operative records were extracted from the original resume. The retrospective cohort used for external validation comprised patients who underwent surgery at Peking University Shenzhen Hospital (cohort 1) and Sun Yat-sen University Cancer Center (cohort 2) during the same period The protocol of this study was approved by the institutional ethics committee of the Sun Yat-sen Memorial Hospital, and consent for the use of data in research was obtained for each participant.

\section{Surgical treatment}

Surgical treatment involved ultrasound-guided vacuumassisted biopsy (UGVAB), lumpectomy, wide local excision, breast-conserving surgery and mastectomy. Ultrasoundguided breast tumor resection is a biopsy procedure for both benign and malignant breast tumor. All of the breast lesions undergoing ultrasound-guided diagnostic breast biopsy were sonographically visible. For the UGVAB procedures, local anesthetic was utilized. After local anesthetic was administered, a \#11 blade was used to make an approximately 5 -mm skin incision entrance. An attempt at complete ultrasound lesion excision was assessed in real-time by taking longitudinal and transverse ultrasound images both during core acquisition and after the completion of core acquisition (12). Little normal breast tissue is resected during this procedure. For those benign tumors can be completely removed by UGVAB, a 
re-excision is not performed. Lumpectomy is the surgical removal of the breast tumor and little normal breast tissue around the lump (width of surgery margin $<1 \mathrm{~cm}$ ). Wide local excision refers to the removal of the breast tumor with a margin of surrounding normal tissue (usually wider than $1 \mathrm{~cm}$ ). Breast-conserving surgery is applied in malignant tumors with complete removal of the tumor and a certain pathological clear margin of normal breast tissue. The surgical margin of UGVAB and lumpectomy was defined as less than $1 \mathrm{~cm}$, wide local excision, breast-conserving surgery and mastectomy was defined $\geq 1 \mathrm{~cm}$. The width of surgical margin is based on the surgery type and surgery record.

\section{Pathological characteristics}

All the original tumor slides were studied. The pathological grade of PTs was diagnosed based on the WHO classification. Five variables were included: stromal cellularity, stromal cell atypia, number of mitoses per 10 high-power field (HPF), stromal overgrowth, and characteristics of the tumor border. The extent of stromal cellularity was based on the overlapping of the nuclei (Figure S1). Stromal overgrowth was defined as the presence of stroma without epithelium in at least one lowpower field, as observed using a $\times 4$ microscope objective. The tumor border was divided into two categories: circumscribed and infiltrative, referring to the absence and presence of projection of tumor stroma into normal breast tissue, respectively (Figure S2). In addition, the presence of necrosis, hemorrhage, and heterogeneous element was also documented.

\section{Follow-up}

All patients were asked to have a subsequent visit 3 months after surgery. For benign and borderline PTs, patients were followed every 12 months. For malignant PTs, patients were followed every 6 months for 5 years and then every 12 months. Detailed recordings of breast examinations were performed at each follow-up visit. Breast ultrasonography, mammograph, chest X-ray and other image examination for suspected organ were performed to detect relapse, distant metastasis or both. For malignant PTs, an extra chest computed tomography (CT) is required annually. Regular follow-up results were obtained from gained from medical records and telephonic interviews. The last follow-up was carried out in July 2018. The follow-up information was gained from medical records and telephonic interviews.

\section{Statistical analysis}

Categorical variables were grouped based on the clinical findings, and decisions on the groups were made before modeling. The results were compared using $\chi^{2}$ test or Fisher's exact test. Continuous variables were compared using $t$-test. Survival curves were depicted using the KaplanMeier method and compared using the log-rank test. Logrank tests and univariate Cox regression were used to screen the risk factors. Cox regression was used for multivariate analysis. Schoenfeld residuals test was used to investigate the proportional hazards assumption (13).

A nomogram was formulated based on the results of multivariate analysis. Bootstraps with 1,000 resamples were used for internal and external validation. Akaike's information criterion was used to screen the variables to avoid overfitting of the model (14). Discrimination ability was assessed using the receiver operating characteristic (ROC) analysis, and predictive accuracy was measured using the concordance index (C-index) reported with its $95 \%$ confidence interval (CI). The $\mathrm{C}$-index was calculated using the Begg's method, which is the modification of Harrel's method and more suitable for Cox regression (15). Larger C-index indicated a more accurate predictive ability of the model. The plot of area under the curve (AUC) change over time was also drawn based on Hung and Chiang's method, which reflected the predictive ability of the model during different time intervals (16). Calibration was evaluated by reviewing the plot of predicted probabilities versus the actual probabilities. The total points according to the established nomogram of each patient were treated as a factor of Cox regression during the external validation of the nomogram (17). The C-index and the slope of the calibration curve were also derived from the regression analysis. A P value $<0.05$ was considered statistically significant. All statistical analysis was done using Stata 13.0 (Stata Corp, 2001; College Station, TX, USA) or R (version 3.2.2, https://www.r-project.org/).

\section{Results}

\section{Patients and clinical outcome}

The primary cohort for model building comprised 334 patients with a median age of 38 years. For the external 
validation cohorts, 36 patients for cohort 1 and 140 patients for cohort 2 were studied. The clinicopathological characteristics of the primary and validation cohorts are listed in Table 1.

Overall, 58 patients had pre-operative biopsy, most $(39 / 58,67.24 \%)$ of the biopsies could not report the histological grade of the tumor, $19(32.76 \%)$ cases were reported with a histological grade, 4 were upgrade and 1 was downgrade after surgery, thus the concordance rate between pre-operative biopsy and surgical excision in histological grade was $73.68 \%(14 / 19)$. The detail were displayed in the Table S1.

The median follow-up was 37 months (range, 15-208 months). The median time of recurrence was 28 months. The 1-, 3-, and 5-year recurrence-free survival was $98.5 \%, 97.9 \%$, and $96.8 \%$ in the primary cohort. The primary cohort had 59 recurrences and 1 lung metastasis and the validation cohort 1 had 9 local recurrences, cohort 2 had 26 local recurrences and 1 lung metastasis.

\section{UGVAB is a non-inferior treatment application in benign $P T$ compared with open surgery}

Of all the 334 patients in primary cohort, 126 (37.72\%) patients had UGVAB. Only 1 (3.7\%) patients in the validation cohort 1 had UGVAB of the 27 patients. No patients in the validation cohort 2 have UGVAB. Thus, $127(24.90 \%)$ patients in our study have been applied with UGVAB.

One hundred and nineteen benign PTs had UGVAB, $14(11.76 \%)$ of them had recurrences. For benign PTs, the UGVAB group was not associated with higher recurrence rate compared with open surgery (lumpectomy \& wide local excision) group [hazard ratio (HR), 1.81; 95\% CI, 0.80-3.98; $\mathrm{P}=0.81]$. After adjusted with other risk factors, UGVAB is still proved to be a non-inferior treatment application (HR, 2.38; 95\% CI, 0.59-9.58; $\mathrm{P}=0.99$ ).

For borderline PTs, 8 cases had UGVAB, $4(50.00 \%)$ of them had recurrences. Compared with 23 (27.71\%) of 83 patients with open surgery (wide local excision and breast conserving surgery), UGVAB is not recommended in treatment of borderline PTs.

\section{Establishment of outcome prediction nomogram}

The results for log-rank tests and univariate Cox regression revealed that the surgery type, surgical margin, mitoses, stromal overgrowth, and tumor border might be the potential risk factors (Tables $S 2, S 3$; all $\mathrm{P}<0.05$ ).

Multivariate Cox regression analysis revealed that the surgical margin, mitoses, and tumor border were independent risk factors for tumor recurrence. The results are presented in Table 2. The results of Schoenfeld residuals test exhibited that the variables involved in the regression analysis and the whole model conformed to the proportional hazards assumption (Table S4). The interaction between surgical margin and tumor border were also analyzed, which demonstrated no significant influence on the whole model $(\mathrm{P}=0.16)$.

The prognostic nomogram based on the results of multivariate Cox regression analysis is illustrated in Figure 1. The C-index of internal validation was 0.71 (95\% CI, 0.67-0.75), while the $\mathrm{C}$-index of external validation cohort 1 was 0.67 (95\% CI, 0.60-0.75) and 0.73 (95\% CI, 0.60-0.83) for cohort 2. The calibration plot of the probability of recurrence-free survival 3 years after surgery is illustrated in Figure $2 A$. The deviations from nomogram predicted survival to nomogram actual survival is all smaller than $10 \%$. Moreover, the calibration curve of external validation showed optimal agreement between the modelpredicted recurrence-free survival and the actual survival (Figure 2B,C). Further, the plot of AUC change over time also showed that the predictive ability of the model was appreciable (Figure S3).

\section{Discussion}

To date, the prognosis of PTs is mainly dependent on the pathological grade. However, this method lacks objectivity similar to other histopathological grades, thereby limiting its application in clinical practice. Besides, words such as mild, moderate, and severe were used to describe some pathological variables, leading to interobserver variability. Lawton et al. reported that the agreement rate in the grading of PTs between pathologists was 53\% (18). Also, a few studies declared that the histological grade was not an independent risk factor for the recurrence of PTs $(4,9,19,20)$. The present study demonstrated no direct correlation between the histological grade and recurrence of PTs. Therefore, a combination of pathological and clinical variables will invariably lead to a more accurate evaluation of the prognosis of PT. Here in, nomograms were established to predict the clinical outcomes of PTs using variables of width of surgical margin, mitoses, and tumor border. These variables will be discussed in detail in the latter part. 
Table 1 Clinicopathological characteristics of patients

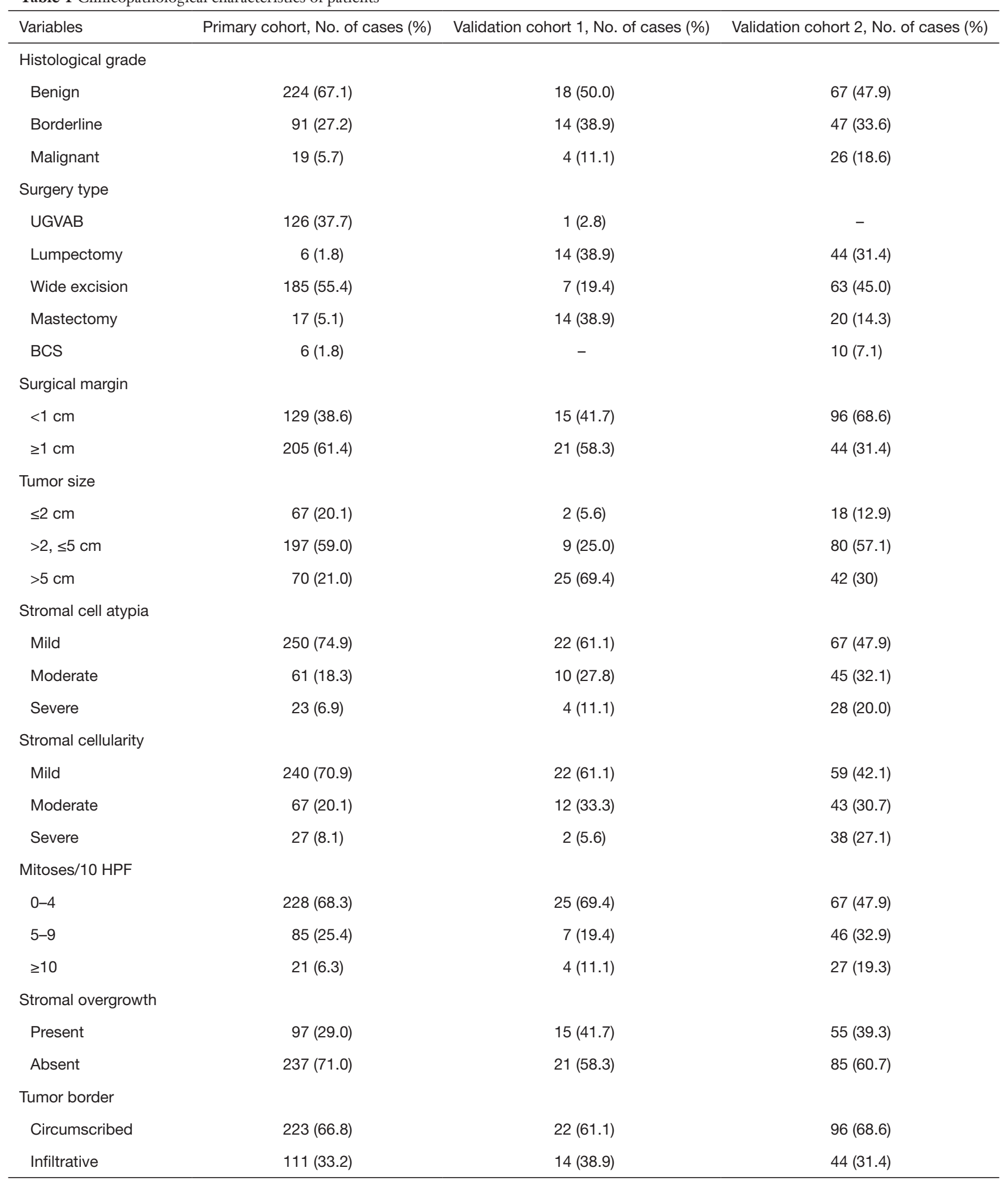

Table 1 (continued) 
Table 1 (continued)

\begin{tabular}{lccc}
\hline Variables & Primary cohort, No. of cases (\%) & Validation cohort 1, No. of cases (\%) & Validation cohort 2, No. of cases (\%) \\
\hline Hemorrhage & $37(11.1)$ & $13(36.1)$ & $11(7.9)$ \\
Present & $297(88.9)$ & $23(63.9)$ & $129(92.1)$ \\
Absent & & & 129 \\
\hline
\end{tabular}

UGVAB, ultrasound-guided vacuum-assisted biopsy; BCS, breast conserving surgery; HPF, high-power field.

Table 2 Results of multivariate Cox regression analysis

\begin{tabular}{lccc}
\hline Variable & Subgroup & HR $(95 \% \mathrm{Cl})$ & $\mathrm{P}$ \\
\hline Surgical margin & $\leq 1 \mathrm{~cm}$ & 1 & - \\
& $>1 \mathrm{~cm}$ & $0.47(0.36-0.63)$ & $<0.001$ \\
Tumor border & Infiltrative & 1 & - \\
& Circumscribed & $0.57(0.39-0.83)$ & 0.003 \\
Mitoses & $0-4$ & 1 & - \\
& $5-9$ & $1.49(1.06-2.08)$ & 0.020 \\
Stromal & $\geq 10$ & $2.01(1.09-3.70)$ & 0.025 \\
overgrowth & Absent & 1 & - \\
& & $1.47(0.95-2.30)$ & 0.085 \\
Surgery type & Present & 1 & - \\
& UGVAB & $0.85(0.56-1.29)$ & 0.792 \\
& Wide excision & $0.58(0.15-2.20)$ & 0.425 \\
& Mastectomy & $0.72(0.16-3.05)$ & 0.657 \\
\hline & BCS & 0.79 &
\end{tabular}

$\mathrm{Cl}$, confidence interval; HR, hazard ratio; UGVAB, ultrasoundguided vacuum-assisted biopsy; BCS, breast conserving surgery.

The characteristics of the tumor border are vital in the grading of PTs. The circumscribed border refers to the tumor growth in a pushing manner, while the infiltrative border refers to the tumor protrusion into the normal breast tissue. A study stated that the tumor border and surgical margin influenced the prognosis synergistically (21). The interaction between tumor border and surgical margin was included as a separate variable into the multivariate analysis in the present study, but no obvious influence was observed on prognosis $(\mathrm{P}=0.16)$. Thus, the surgical margin and tumor border were still independent risk factors for recurrencefree survival.

However, debate continues on the surgery type and surgical margin in the treatment of PTs. It is usually difficult to diagnose PTs prior to operation due to their special pathological characteristics. The reported consistency between the preoperational biopsy (core needle biopsy and fine needle aspiration) and post operational pathological results is about $50-60 \%$ (22-24). The accuracy of intraoperative frozen section examination in PTs is also limited.

UGVAB is not only a biopsy method but also an alternative treatment for small breast mass. It is used extensively in clinical practice because it causes minimal injury. A retrospective study involving 225 patients stated that no difference existed in the recurrence-free survival of patients treated with UGVAB and open surgery (HR, 0.34; 95\% CI, 0.08-1.43) (25). For benign PTs, our study also proved that UGVAB is an effective treatment procedure (HR, 1.81; 95\% CI, 0.80-3.98) The surgical margin status (positive or negative) of benign PTs is always neglected in the clinical practice. Previous studies also demonstrated that surgical margin status has no correlation with recurrence rate in benign PTs $(26,27)$. Thus, UGVAB is an effective biopsy and treatment procedure in benign PTs with uncertain surgical margin status. However, a retrospective study involving 285 patients pointed out that a wide excision and a clear margin of $1 \mathrm{~cm}$ should be ascertained in small tumors (tumor size $<5 \mathrm{~cm}$ ) with frequent mitoses $(>10 / 10$ HPF), even with a second surgery (28). In the present study, the univariate analysis (log-rank test and Cox regression) revealed that the surgery type influenced the recurrencefree survival. However, this trend became inconspicuous after adjusting pathological variables and surgical margin. These results suggested that a second surgery was not necessary for benign PTs treated with UGVAB especially those with fewer mitoses and circumscribed border.

For borderline and malignant PTs, mastectomy is better than breast-conserving surgery to avoid recurrence (29). Some surgeons even consider mastectomy for all borderline and malignant PTs (30). However, a retrospective study involving 81 malignant $\mathrm{PTs}$ indicated no difference in 

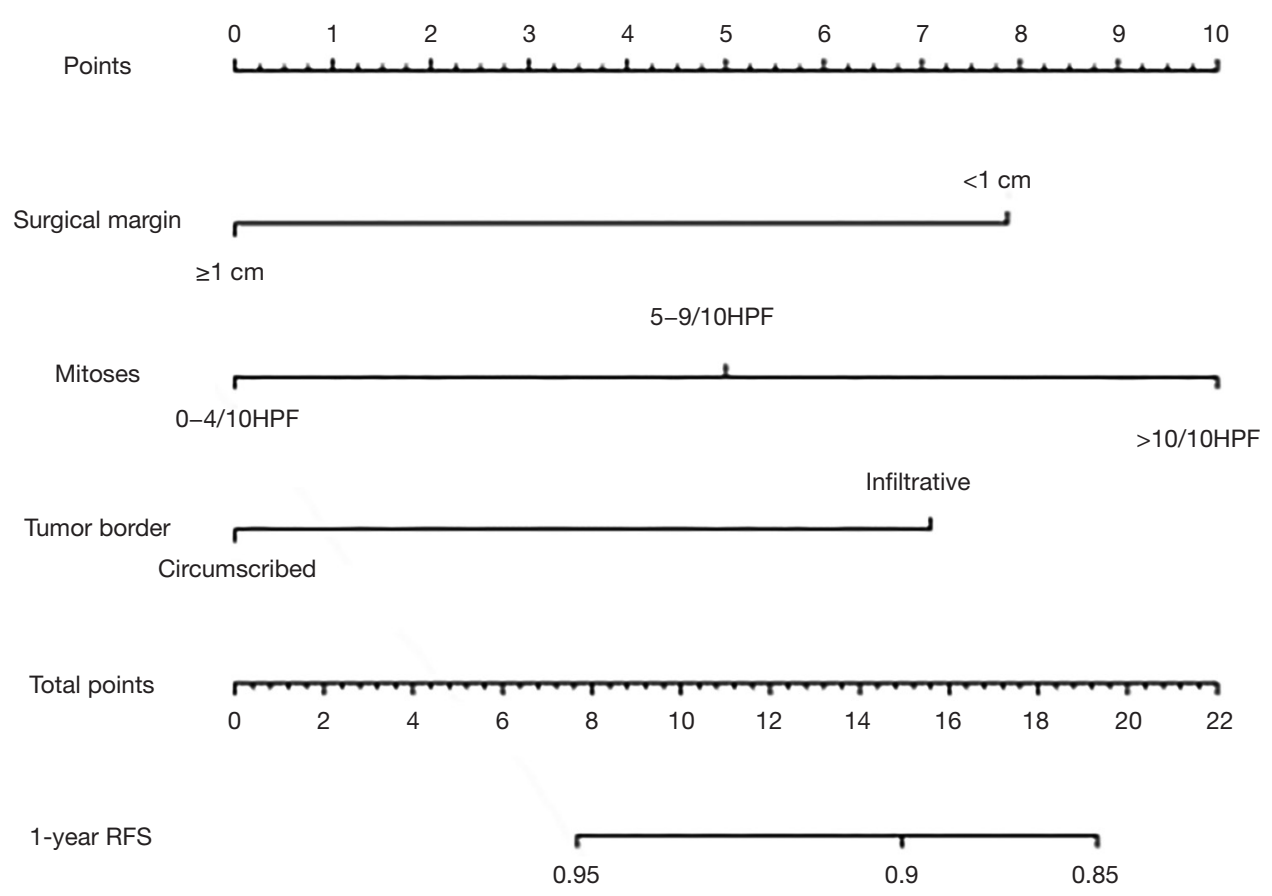

2-year RFS

$\begin{array}{lllllll}0.9 & 0.85 & 0.8 & 0.75 & 0.7 & 0.6 & 0.5\end{array}$

3-year RFS

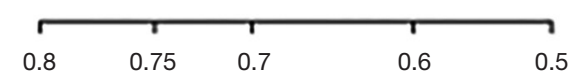

Figure 1 Nomograms for predicting recurrence-free survival (RFS). A nomogram predicting 1-, 2-, and 3-year RFS probabilities of patients with phyllodes tumors in Sun Yat-sen Memorial Hospital training set. Points were assigned for width of surgical margin, mitoses and tumor border, by drawing a line upward from the corresponding values to the "Points" line. The sum of these three points, plotted on the "Total Points" line, corresponds to the prediction of probability of 1-, 2-, and 3-year RFS probabilities.

disease-related deaths between mastectomy and breastconserving surgery (1). Other studies also stated that breast-conserving surgery with negative margin was not inferior to mastectomy $(31,32)$. The present study involved 110 patients with borderline and malignant PTs, among which 17 patients had a mastectomy and 93 had breastconserving surgery or wide excision. No significant difference was found in the recurrence-free survival between these two surgery types (HR, 1.04; 95\% CI, 0.54-2.02; $\mathrm{P}=0.89$ ).

The mainstay of the surgical treatment in PTs is a guarantee of the negative surgical margin due to the leaflike architecture and the unclear border of PTs $(19,33,34)$. Some surgeons insisted that the surgical margin should be wider than $1 \mathrm{~cm}$. Recent studies suggested that it was unnecessary for all PTs to have such wide excision. The extent of excision was not correlated with the recurrence of PTs, while it emphasized the guarantee of clear surgical margins (21,35). Nevertheless, the results of this study supported that wider surgical margin was beneficial for a recurrence-free survival (HR, 0.50; 95\% CI, 0.38-0.65). This trend was still strong after adjusting the pathological variable and surgery type. Thus, adequate surgical intervention is of great importance in preventing recurrence of PTs.

Nomograms combining clinical and pathological variables have been proved to be better than stage grouping. For example, a young patient had UGVAB for breast lump. The pathological diagnosis was benign PT. Thus, we don't have special follow-up schedule for the patient. However, 
A

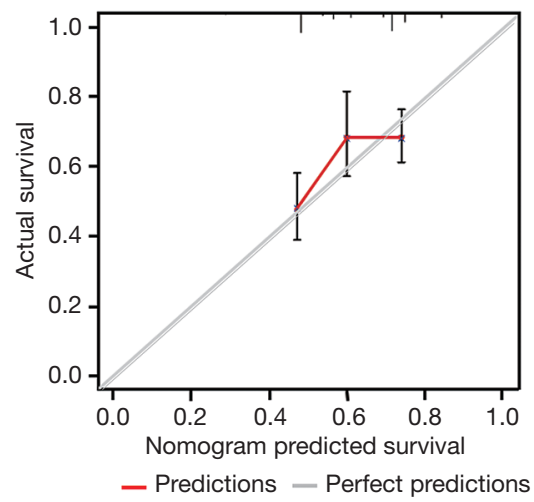

B

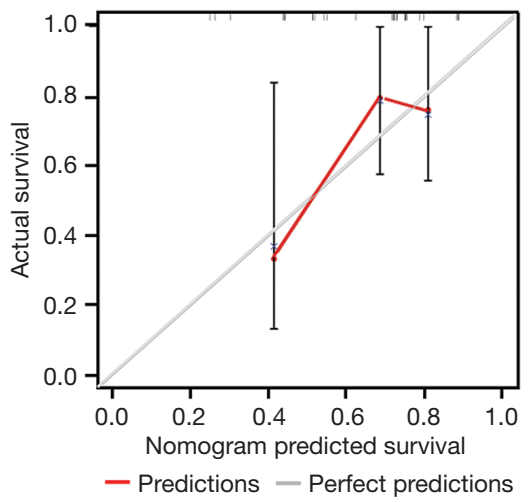

C

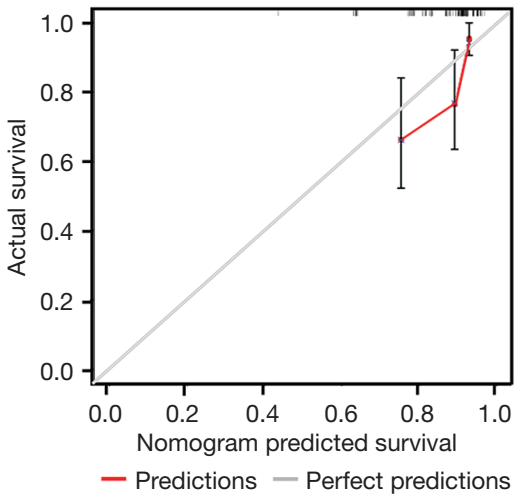

Figure 2 Calibration of the nomogram in training and validation cohorts. The $\mathrm{x}$-axis represents nomogram-predicted recurrence-free survival, and the y-axis represents actual recurrence-free survival, with 95\% confidential intervals measured by Kaplan-Meier analysis. A represents the 3-year survival of the primary training set of Sun Yat-sen Memorial Hospital; B represents validation cohort 1 of Peking University Shenzhen Hospital; C represents validation cohort 2 of Sun Yat-sen University Cancer Center.

if we calculate based on our nomogram, the follow-up schedule may be changed. She had UGVAB, thus the surgical margin was less than $1 \mathrm{~cm}$ (get 8 points for surgical margin parameter), the mitoses counts were 6/10 HPF (get 5 points for mitoses parameter) and circumscribed border (get 0 points for border parameter), thus the total points for the patients is 13 . The 1-, 2-, and 3-year predicted recurrence-free survival was $90-95 \%, 70-75 \%$, and $50-55 \%$ based on our nomogram. Thus, we may suggest the patient to have a re-excision to get a wider margin or follow up the patient closely instead.

The AMOS criteria have also been proved to be better than the histological score system of PTs (7). The prediction model based on the width of surgical margin, mitoses, and tumor border showed good prediction ability (C-index, 0.71; 95\% CI, 0.67-0.75). It also showed optimal prediction ability during external validation (cohort $1 \mathrm{C}$-index, 0.67 , 95\% CI, 0.60-0.74; cohort $20.73,95 \%$ CI, 0.60-0.83). The AMOS criteria are the only published prediction model of PTs. It showed excellent prediction ability in the primary cohort (C-index, 0.79$)$ and the external validation cohort (C-index, 0.90) (36). However, we are unable to use this model due to that most of the surgical margin status (negative or positive) of all the benign and some borderline PTs were unknown in our cohorts.

The present study had several limitations. First, the number of model building cohort and validation cohort was limited. The model needs to be validated using larger samples and different centers. Second, the number of malignant PTs included during model development was limited, making the prediction ability in malignant PTs crude. Thus, this model was more suitable for evaluating the prognosis of benign and borderline PTs. Besides, the diagnosis and grading of PTs mostly relied on the histopathological characteristics. Therefore, specific immunohistochemical markers for PTs are urgently needed. The accuracy of the model can be greatly improved by adding some specific immunohistochemical markers.

\section{Conclusions}

Nowadays, the surgical margin status (positive or negative) of benign and some borderline PTs is always neglected in clinical practice. We have built a prediction model based on width of surgical margin, which can be easily applied in clinical practice. The prediction model with a combination of multiple clinicopathological variables can be a useful adjuvant tool for making clinical decisions and selecting the treatment and follow-up schedule after surgery. It can be widely applied in clinical practice helping decide re-excision or "wait and watch".

\section{Acknowledgments}

We thank all the patient who participated in this study and for their willingness to contribute valuable data for this manuscript.

Funding: This study was supported by grants from 
the National Science and Technology Major Project (No. 2020ZX09201021), the National Natural Science Foundation of China (No. 81872158, 81572596 , 81972471, U1601223), the Natural Science Foundation of Guangdong Province (No. 2017A030313828), Elite Young Scholars Program of Sun Yat-Sen Memorial Hospital (No. Y201703). Yat-Sen Scholarship for Young Scientist, the Guangzhou Science and Technology Major Program (No. 201704020131), the Sun Yat-Sen University Clinical Research 5010 Program (No. 2018007), the Sun Yat-Sen Clinical Research Cultivating Program (No. SYS-C-201801), the Guangdong Science and Technology Department (No. 2017B030314026), the Medical Artificial Intelligence Project of Sun Yat-Sen Memorial Hospital (No. YXRGZN201902). Grant KLB09001 from the Key Laboratory of Malignant Tumor Gene Regulation and Target Therapy of Guangdong Higher Education Institutes, Sun Yat-Sen University and Grant [2013]163 from Key Laboratory of Malignant Tumor Molecular Mechanism and Translational Medicine of Guangzhou Bureau of Science and Information Technology. Guangdong Science and Technology Department (No. 2017B030314026). The Medical Artificial Intelligence Project of Sun Yat-Sen Memorial Hospital (No. YXRGZN201902).

\section{Footnote}

Conflicts of Interest: The authors have no conflicts of interest to declare.

Ethical Statement: The authors are accountable for all aspects of the work in ensuring that questions related to the accuracy or integrity of any part of the work are appropriately investigated and resolved. The protocol of this study was approved by the institutional ethics committee of the Sun Yat-sen Memorial Hospital (No. SYSEC-KY-KS-2018-019), and consent for the use of data in research was obtained for each participant.

Open Access Statement: This is an Open Access article distributed in accordance with the Creative Commons Attribution-NonCommercial-NoDerivs 4.0 International License (CC BY-NC-ND 4.0), which permits the noncommercial replication and distribution of the article with the strict proviso that no changes or edits are made and the original work is properly cited (including links to both the formal publication through the relevant DOI and the license). See: https://creativecommons.org/licenses/by-nc-nd/4.0/.

\section{References}

1. Macdonald OK, Lee CM, Tward JD, et al. Malignant phyllodes tumor of the female breast: association of primary therapy with cause-specific survival from the Surveillance, Epidemiology, and End Results (SEER) program. Cancer 2006;107:2127-33.

2. Lakhani SR, Ellis IO, Schnitt SJ, et al. WHO Classification of Tumours of the Breast. WHO Classification of Tumours, 4th edition, Volume 4, 2012.

3. Zhang Y, Kleer CG. Phyllodes Tumor of the Breast: Histopathologic Features, Differential Diagnosis, and Molecular/Genetic Updates. Arch Pathol Lab Med 2016;140:665-71.

4. Barrio AV, Clark BD, Goldberg JI, et al. Clinicopathologic features and long-term outcomes of 293 phyllodes tumors of the breast. Ann Surg Oncol 2007;14:2961-70.

5. Briggs RM, Walters M, Rosenthal D. Cystosarcoma phylloides in adolescent female patients. Am J Surg 1983;146:712-4.

6. Parker SJ, Harries SA. Phyllodes tumours. Postgrad Med J 2001;77:428-35.

7. Tan PH, Thike AA, Tan WJ, et al. Predicting clinical behaviour of breast phyllodes tumours: a nomogram based on histological criteria and surgical margins. J Clin Pathol 2012;65:69-76.

8. Cohn-Cedermark G, Rutqvist LE, Rosendahl I, et al. Prognostic factors in cystosarcoma phyllodes. A clinicopathologic study of 77 patients. Cancer 1991;68:2017-22.

9. Gnerlich JL, Williams RT, Yao K, et al. Utilization of radiotherapy for malignant phyllodes tumors: analysis of the National Cancer Data Base, 1998-2009. Ann Surg Oncol 2014;21:1222-30.

10. Sawalhi S, Al-Shatti M. Phyllodes tumor of the breast: a retrospective study of the impact of histopathological factors in local recurrence and distant metastasis. Ann Saudi Med 2013;33:162-8.

11. Taira N, Takabatake D, Aogi K, et al. Phyllodes tumor of the breast: stromal overgrowth and histological classification are useful prognosis-predictive factors for local recurrence in patients with a positive surgical margin. Jpn J Clin Oncol 2007;37:730-6.

12. Povoski SP, Jimenez RE. A comprehensive evaluation of the 8 -gauge vacuum-assisted Mammotome(R) system for ultrasound-guided diagnostic biopsy and selective excision of breast lesions. World J Surg Oncol 2007;5:83.

13. Grambsch PM, Therneau TM. Proportional hazards tests 
and diagnostics based on weighted residuals. Biometrika 1994;81:515-26.

14. Steyerberg EW, Harrell FE Jr, Borsboom GJ, et al. Internal validation of predictive models: efficiency of some procedures for logistic regression analysis. J Clin Epidemiol 2001;54:774-81.

15. Begg CB, Cramer LD, Venkatraman ES, et al. Comparing tumour staging and grading systems: a case study and a review of the issues, using thymoma as a model. Stat Med 2000;19:1997-2014.

16. Hung H, Chiang CT. Estimation methods for timedependent AUC models with survival data. Can J Statistics 2010;38:8-26.

17. Balachandran VP, Gonen M, Smith JJ, et al. Nomograms in oncology: more than meets the eye. Lancet Oncol 2015;16:e173-80.

18. Lawton TJ, Acs G, Argani P, et al. Interobserver variability by pathologists in the distinction between cellular fibroadenomas and phyllodes tumors. Int J Surg Pathol 2014;22:695-8.

19. Guillot E, Couturaud B, Reyal F, et al. Management of phyllodes breast tumors. Breast J 2011;17:129-37.

20. Barth RJ Jr, Wells WA, Mitchell SE, et al. A prospective, multi-institutional study of adjuvant radiotherapy after resection of malignant phyllodes tumors. Ann Surg Oncol 2009;16:2288-94.

21. Jang JH, Choi MY, Lee SK, et al. Clinicopathologic risk factors for the local recurrence of phyllodes tumors of the breast. Ann Surg Oncol 2012;19:2612-7.

22. Tsang AK, Chan SK, Lam CC, et al. Phyllodes tumours of the breast - differentiating features in core needle biopsy. Histopathology 2011;59:600-8.

23. Choi J, Koo JS. Comparative study of histological features between core needle biopsy and surgical excision in phyllodes tumor. Pathol Int 2012;62:120-6.

24. El Hag IA, Aodah A, Kollur SM, et al. Cytological clues in the distinction between phyllodes tumor and fibroadenoma. Cancer Cytopathol 2010;118:33-40.

25. Ouyang Q, Li S, Tan C, et al. Benign Phyllodes Tumor of the Breast Diagnosed After Ultrasound-Guided Vacuum-

Cite this article as: Chao X, Jin X, Tan C, Sun P, Cui J, Hu H, Ouyang Q, Chen K, Wu W, He Z, Nie Y, Yao H. Re-excision or "wait and watch" - a prediction model in breast phyllodes tumors after surgery. Ann Transl Med 2020;8(6):371. doi: 10.21037/atm.2020.02.26
Assisted Biopsy: Surgical Excision or Wait-and-Watch? Ann Surg Oncol 2016;23:1129-34.

26. Cowan ML, Argani P, Cimino-Mathews A. Benign and low-grade fibroepithelial neoplasms of the breast have low recurrence rate after positive surgical margins. Mod Pathol 2016;29:259-65.

27. Teo JY, Cheong CS, Wong CY. Low local recurrence rates in young Asian patients with phyllodes tumours: less is more. ANZ J Surg 2012;82:325-8.

28. Yom CK, Han W, Kim SW, et al. Reappraisal of conventional risk stratification for local recurrence based on clinical outcomes in 285 resected phyllodes tumors of the breast. Ann Surg Oncol 2015;22:2912-8.

29. Ben Hassouna J, Damak T, Gamoudi A, et al. Phyllodes tumors of the breast: a case series of 106 patients. Am J Surg 2006;192:141-7.

30. Chaney AW, Pollack A, McNeese MD, et al. Primary treatment of cystosarcoma phyllodes of the breast. Cancer 2000;89:1502-11.

31. Moutte A, Chopin N, Faure C, et al. Surgical Management of Benign and Borderline Phyllodes Tumors of the Breast. Breast J 2016;22:547-52.

32. Mituś J, Reinfuss M, Mitus JW, et al. Malignant phyllodes tumor of the breast: treatment and prognosis. Breast J 2014;20:639-44.

33. Lavoué V, Fritel $\mathrm{X}$, Antoine $M$, et al. Clinical practice guidelines from the French College of Gynecologists and Obstetricians (CNGOF): benign breast tumors - short text. Eur J Obstet Gynecol Reprod Biol 2016;200:16-23.

34. Bhargav PR, Mishra A, Agarwal G, et al. Phyllodes tumour of the breast: clinicopathological analysis of recurrent vs. non-recurrent cases. Asian J Surg 2009;32:224-8.

35. Lin CC, Chang HW, Lin CY, et al. The clinical features and prognosis of phyllodes tumors: a single institution experience in Taiwan. Int J Clin Oncol 2013;18:614-20.

36. Nishimura R, Tan PH, Thike AA, et al. Utility of the Singapore nomogram for predicting recurrence-free survival in Japanese women with breast phyllodes tumours. J Clin Pathol 2014;67:748-50. 


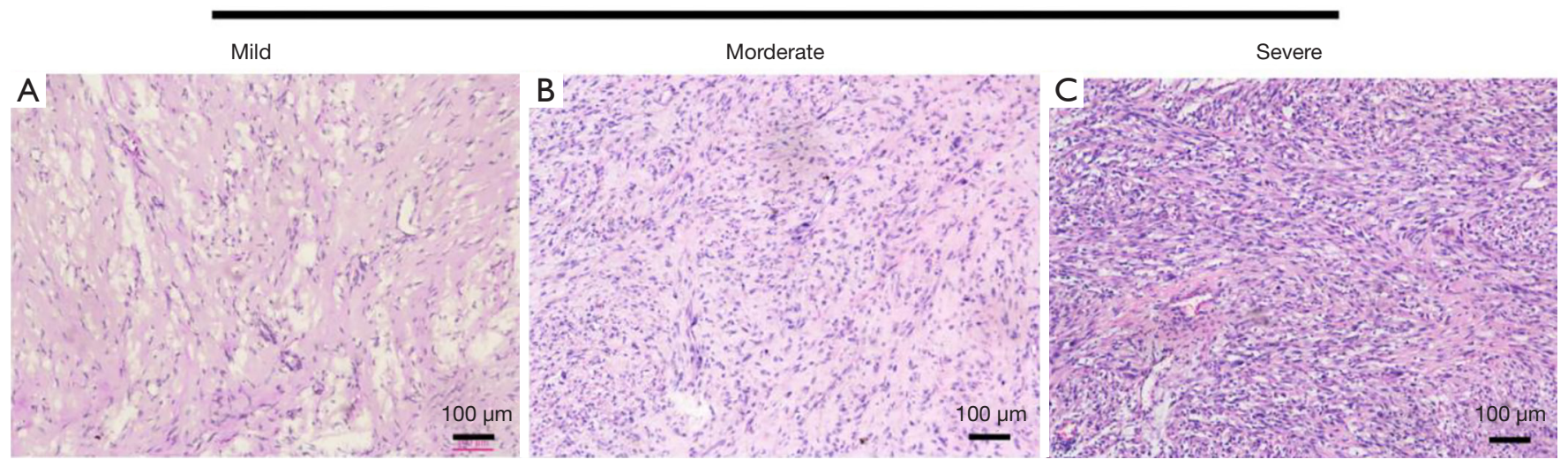

Figure S1 Stromal cellularity has to be evaluated in most cellular areas (HE staining): (A) mild: twice cellularity of normal perilobular stroma without nuclei overlapping, (B) moderate: intermediate degree between mild severe, and (C) severe: stromal cells in close contiguity with nuclei appearing to touch and overlapping.

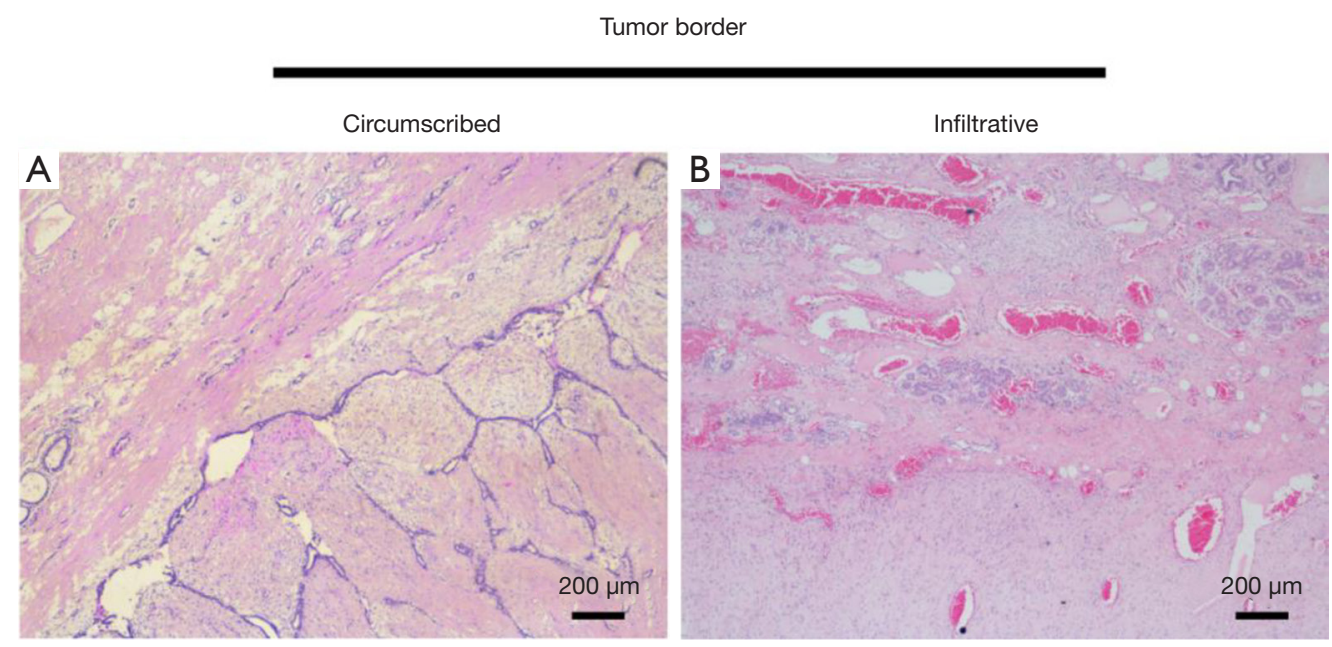

Figure S2 Tumor border: (A) circumscribed: a pushing border without tumor protruding to peritumor tissue and (B) infiltrative: projections of tumor into peritumor tissue without a clear border. 
Table S1 Characteristics of pre-operative pathological diagnosis

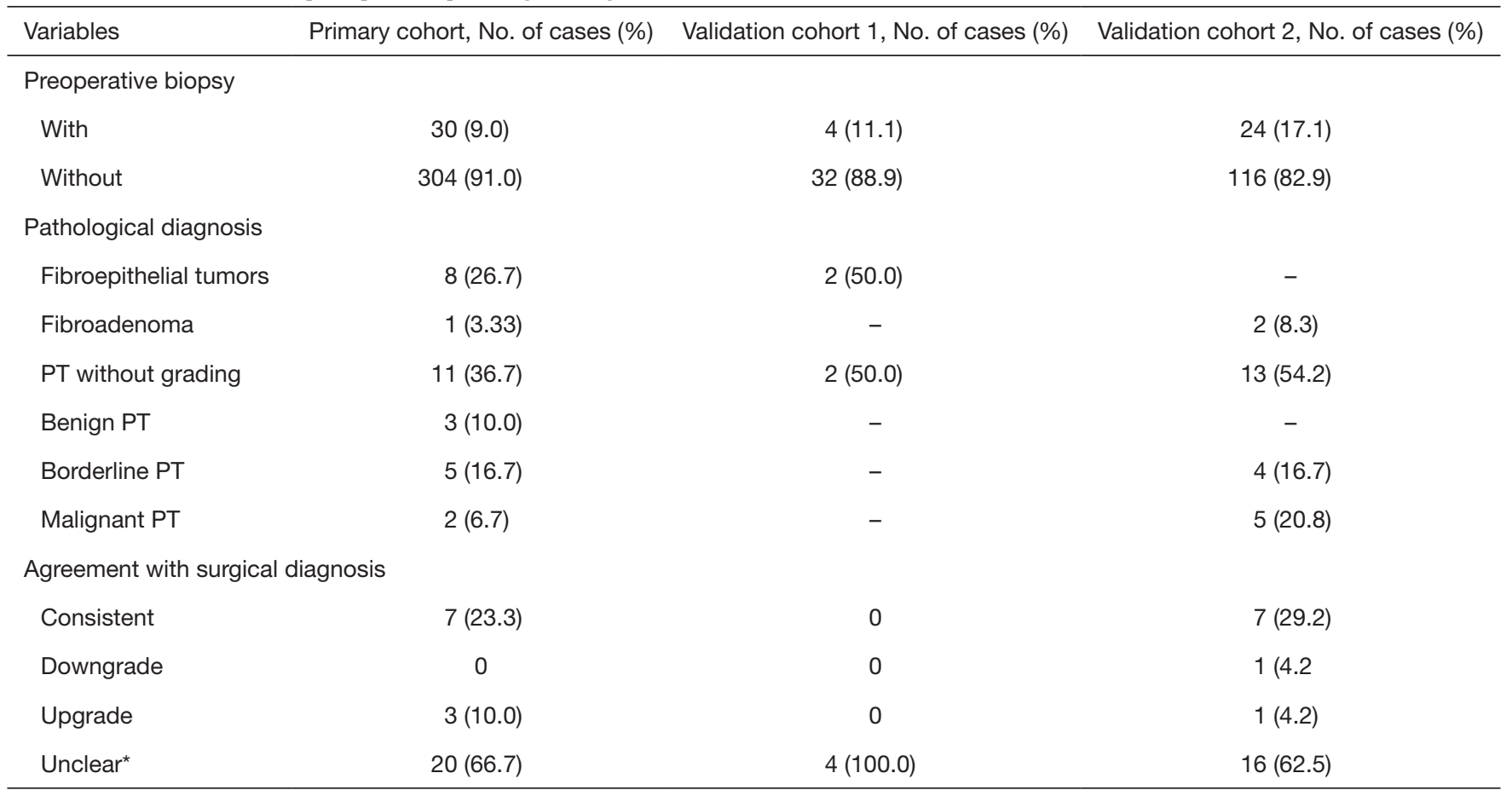

Unclear* refers to those pre-operative diagnosis without specific histological grade. PT, phyllodes tumor. 
Table S2 Results of log-rank test

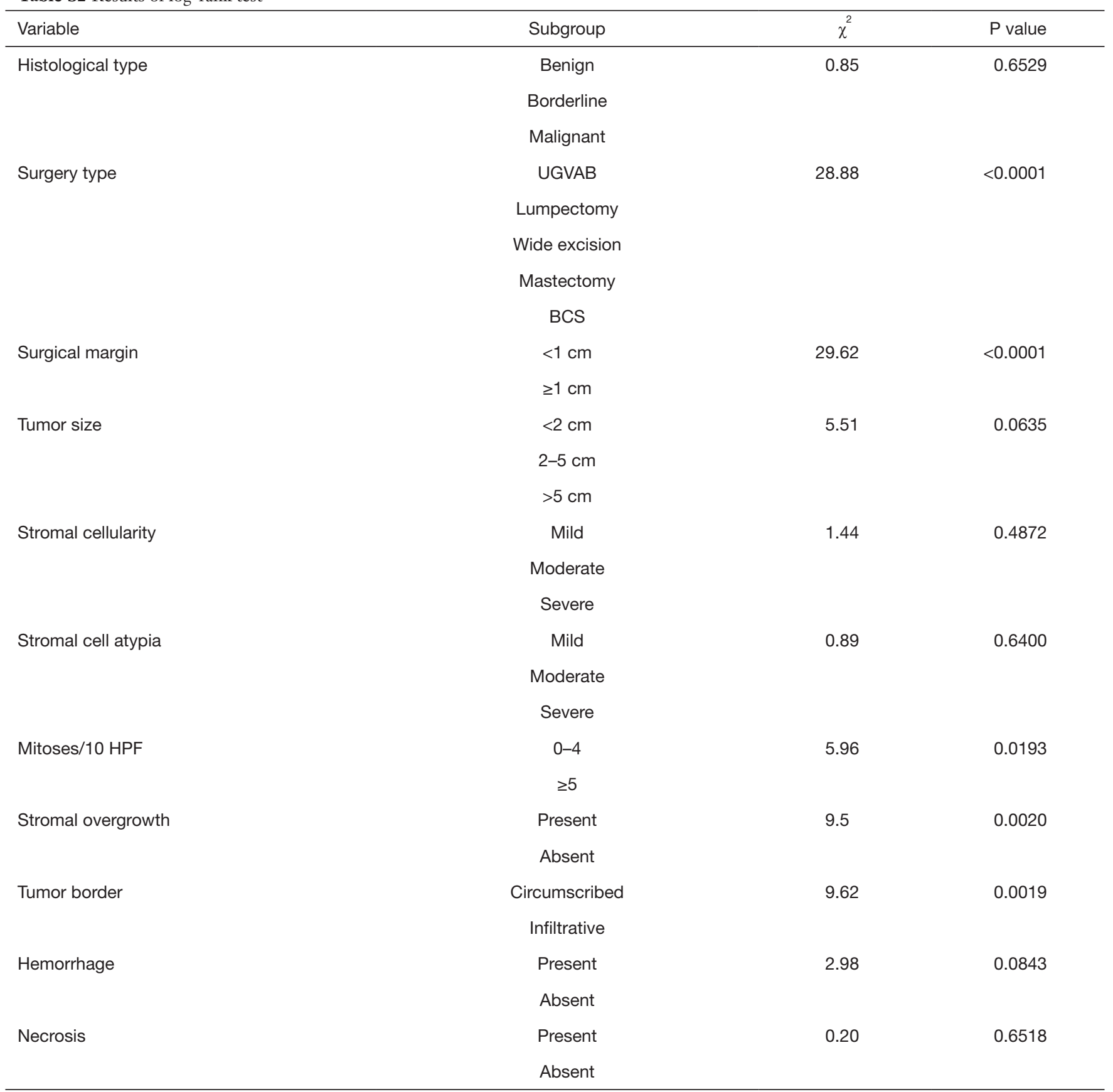

UGVAB, ultrasound-guided vacuum-assisted biopsy; BCS, breast conserving surgery; HPF, high-power field. 
Table S3 Results of univariate Cox regression analysis

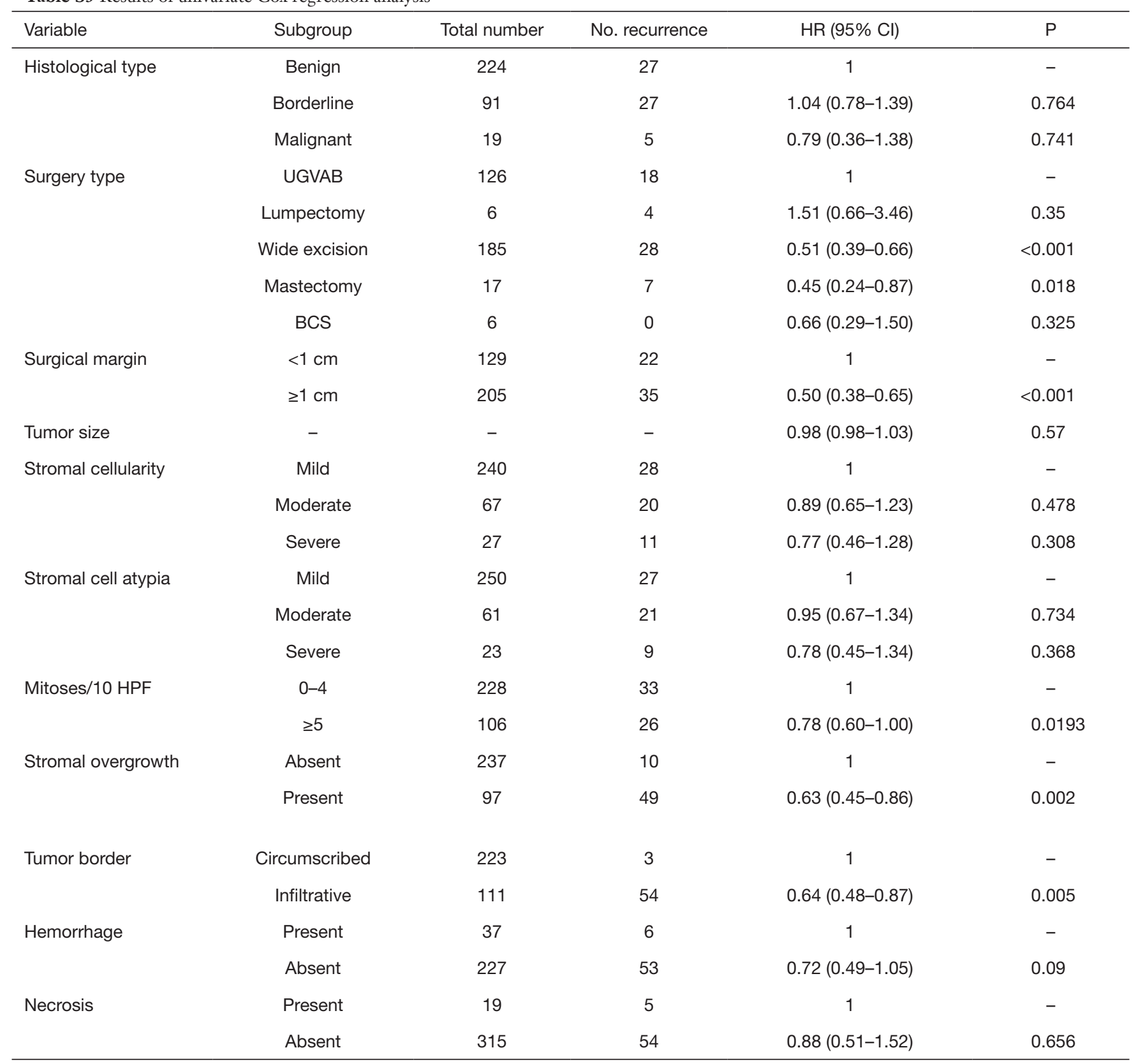

HR, hazard ratio; $\mathrm{Cl}$, confidence interval; UGVAB, ultrasound-guided vacuum-assisted biopsy; BCS, breast conserving surgery; HPF, highpower field. 
Table S4 Cox proportional hazards assumption

\begin{tabular}{lcl}
\hline Variables & $\chi^{2}$ & P value \\
\hline Surgical margin & 0.04 & 0.8340 \\
Tumor border & 0.45 & 0.5015 \\
Mitoses & 0.52 & 0.4690 \\
Overall & 0.78 & 0.8544 \\
\hline
\end{tabular}

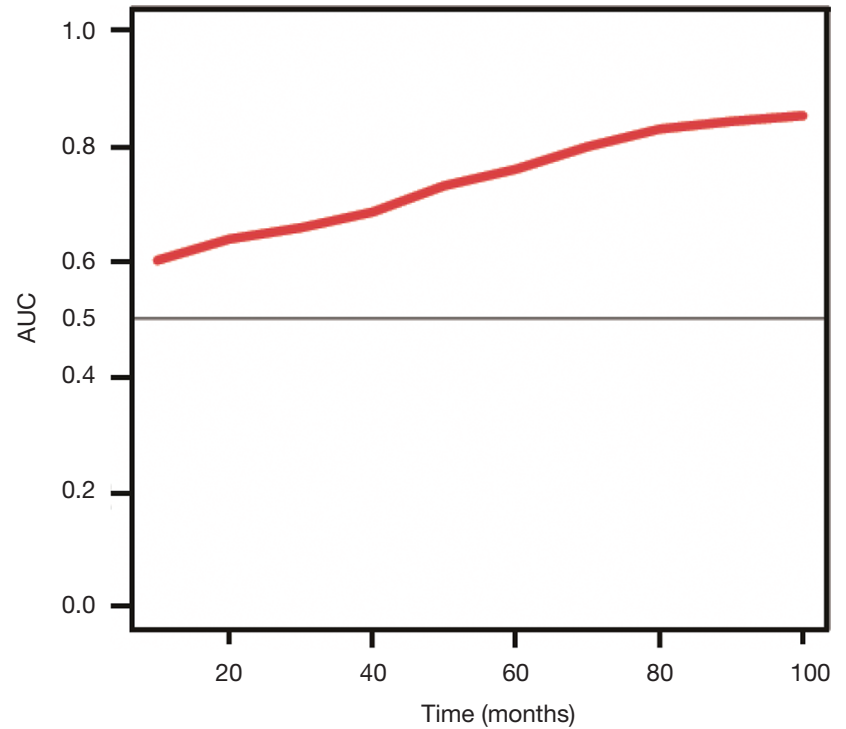

Figure S3 AUC of the nomogram change over time. AUC, area under the curve. 